\title{
FURTHER PROPERTIES OF THE NONSEPARABLE SOLUTIONS OF THE HELMHOLTZ WAVE EQUATION*
}

\author{
BY \\ DONALD S. MOSELEY \\ Vitro Laboratories, Silver Spring, Maryland
}

\begin{abstract}
Nonseparable solutions $W^{(n)}$ of $\left(\nabla^{2}+k^{2}\right) W^{(n)}=0$ are linearly independent, but inter-related through a generative differential operator. The nonseparable of order $n=0$ is the familiar separable solution. In two cartesian coordinates, a sum of zero and second order solutions can describe transverse motion of a membrane of unique boundary contour. In three coordinates the same sum can describe acoustic pressure in a uniquely shaped cavity with pressure-release walls.
\end{abstract}

Introduction. Conventional solutions of the Helmholtz or time-reduced wave equation are readily found through the college-taught method of separation of variables. However, there exist other independent solutions, derivable from these (as will be shown) yet not obtainable by this method. The latter are being called nonseparable solutions, because none is a simple product of functions, and hence none can be separated into factors that contain one independent variable in each. This behavior was shown in a previous paper [1], which presented nonseparable solutions of the vector Helmholtz wave equation in circular cylindrical coordinates limited to symmetry about the axis.

The Helmholtz wave equation in scalar form is

$$
\left(\nabla^{2}+k^{2}\right) W=0
$$

where $\nabla^{2}$ is the Laplacian operator for the appropriate coordinate system, $k$ is the phase constant, $\omega / c$, and $W$ is the solution.

In all that follows, rectangular coordinates will be used, so that the Laplacian is

$$
\nabla^{2}=\partial^{2} / \partial x^{2}+\partial^{2} / \partial y^{2}+\partial^{2} / \partial z^{2}
$$

Two-coordinate variables. Let $W=f(x, y)$. The separable solution is then

$$
W=\sin (a x+\alpha) \sin (b y+\beta)
$$

where

$$
k^{2}=a^{2}+b^{2}
$$

and $\alpha$ and $\beta$ are constants.

If we form the two-dimensional operator

$$
O_{2 C}=b \partial / \partial a-a \partial / \partial b
$$

and operate upon the solution $W$, we obtain a nonseparable function which is likewise a solution of (1), when (4) is true. Operating a second time, we obtain a second non-

* Received March 1, 1968; revised version received September 22, 1968. The major portion of this work was supported by the U. S. Army Aviation Materiel Laboratories, Fort Eustis, Virginia, under Contract DA 44-177-AMC-342(T). 
separable solution. Indeed, the iterative use of $O_{2 C}$, as a generative operator, generalizes to

$$
W^{(n+1)}=O_{2 C} W^{(n)}, \quad n=0,1,2,3 \cdots,
$$

provided we consider $W$ of Eq. (3) to be $W^{(0)}$, a nonseparable solution of zero order. ${ }^{1}$

Basically the above is not different from considering that $W=W(x, y, a, b)$, where $b=b(a)$ by Eq. (4); and Eq. (6) can be written as

$$
W^{(n+1)}=b(\partial / \partial a) b=W^{(n)}
$$

since $a \neq a(x, y)$.

The first four members of the set of nonseparable solutions based on Eq. (3) are

$$
\begin{aligned}
& W^{(0)}=\sin (a x+\alpha) \sin (b y+\beta) \\
& W^{(1)}=b x \cos (a x+\alpha) \sin (b y+\beta)-a y \sin (a x+\alpha) \cos (b y+\beta) \\
& W^{(2)}=-\left\{\left[(b x)^{2}+(a y)^{2}\right] \sin (a x+\alpha) \sin (b y+\beta)+b y \sin (a x+\alpha) \cos (b y+\beta)\right. \\
& \quad+a x \cos (a x+\alpha) \sin (b y+\beta)+2 a b x y \cos (a x+\alpha) \cos (b y+\beta)\} \\
& \begin{aligned}
W^{(3)}=\left[3(b x)^{2}+(a y)^{2}+\right. & 1] a y \sin (a x+\alpha) \cos (b y+\beta) \\
& +3 a b\left(x^{2}-y^{2}\right) \sin (a x+\alpha) \sin (b y+\beta) \\
& +3 x y\left(a^{2}-b^{2}\right) \cos (a x+\alpha) \cos (b y+\beta) \\
& -\left[3(a y)^{2}+(b x)^{2}+1\right] b x \cos (a x+\alpha) \sin (b y+\beta)
\end{aligned}
\end{aligned}
$$

where, for each $W^{(n)}$, the frequency equation is Eq. (4).

Another two-dimensional set can be derived from the exponential form of separable solution, namely, $W=V(r, s)=\exp i r$. Let

$$
r=a x \pm b y
$$

and

$$
s=b x \mp a y .
$$

Then the generative operator becomes

$$
O_{2 C}=s \partial / \partial r-r \partial / \partial s
$$

and the solutions of order $0 \leq n \leq 5$ are

$$
\begin{aligned}
& V^{(0)}=e^{i r}, \\
& V^{(1)}=i s e^{i r}, \\
& V^{(2)}=\left[(i s)^{2}-i r\right] e^{i r}, \\
& V^{(3)}=i s\left[(i s)^{2}-3 i r-1\right] e^{i r}, \\
& V^{(4)}=\left[(i s)^{4}-6 i r(i s)^{2}+3(i r)^{2}-4(i s)^{2}+i r\right] e^{i r}, \\
& V^{(5)}=i s\left[(i s)^{4}-10 i r(i s)^{2}+15(i r)^{2}-10(i s)^{2}+15 i r+1\right] e^{i r} .
\end{aligned}
$$

Members of this set are related to $W=f(x, y)$ through the following expression:

$$
\operatorname{Re}\left[V_{-}^{(n)}-V_{+}^{(n)}\right]=2 W^{(n)},
$$

1 "Order" and "operator" are applicable to solutions in [1]. See Appendix. 
where the plus and minus subscripts indicate which choice of algebraic sign has been taken in $r$.

Three-coordinate variables. With reference to Eq. (1), let $W=f(x, y, z)$. The separable solution is then

$$
W^{(0)}=\sin u \sin v \sin w
$$

where

where

$$
u=a x+\alpha, \quad v=b y+\beta, \quad w=d z+\delta,
$$

$$
k^{2}=a^{2}+b^{2}+d^{2}
$$

and $\delta$ is a constant.

The generative operator for obtaining nonseparable solutions is not uniquely defined in three dimensions, since $W=W(x, y, z, a, b, d)$, where $b=b(a, d)$, by Eq. (24).

One operator $O_{3 c}$ has been worked out for the equation

$$
W^{(n+1)}=O_{3 c} W^{(n)}
$$

and it is

$$
O_{3 c}=f \partial / \partial a-g \partial / \partial b-h \partial / \partial d
$$

where

$$
f=b+d, \quad g=a+d, \quad h=a-b .
$$

Nonseparable solutions of Eq. (1), derived with the aid of Eq. (25), are $W^{(0)}=\sin u \sin v \sin w$,

$W^{(1)}=f x \cos u \sin v \sin w-g y \sin u \cos v \sin w-h z \sin u \sin v \cos w$, $W^{(2)}=\left[(f x)^{2}+(g y)^{2}+(h z)^{2}\right] \sin u \sin v \sin w$

$+(g+h) x \cos u \sin v \sin w-2 g h y z \sin u \cos v \cos w$

$+(f-h) y \sin u \cos v \sin w+2 f h x z \cos u \sin v \cos w$

$+(f+g) z \sin u \sin v \cos w+2 f g x y \cos u \cos v \sin w$,

$W^{(3)}=\cdots$.

and

$$
\begin{aligned}
W^{(1)}= & x \sin u(b \cos v \sin w-d \sin v \cos w) \\
& +y \sin v(d \sin u \cos w-a \cos u \sin w) \\
& +z \sin w(a \cos u \sin v-b \sin u \cos v),
\end{aligned}
$$

$$
\begin{aligned}
W^{(2)}= & \left(x^{2}+y^{2}+z^{2}\right) \sin x \sin y \sin z \\
& +x \cos x \sin y \sin z+y \sin x \cos y \sin z \\
& +z \sin x \sin y \cos z+y z \sin x \cos y \cos z \\
& +x z \cos x \sin y \cos z+x y \cos x \cos y \sin z .
\end{aligned}
$$


Eq. (32) is a symmetric function, since it transforms into itself when its variables are interchanged in pairs.

Application to membranes. The simplest two-dimensional application of the Helmholtz wave equation is to membrane motion at right angles to the plane of a membrane. If $\eta$ be defined as the instantaneous displacement of any point of a membrane, then $W^{(n)}$, of the set illustrated by Eqs. (8-11), may be regarded as the amplitude of the displacement in the equation

$$
\eta=W^{(n)} \cos \omega t .
$$

Zero amplitude, the customary Dirichlet condition for a membrane boundary, occurs at all points $\left(x_{i}, y_{i}\right)$ which solve the two-dimensional transcendental equation

$$
W^{(n)}=0 .
$$

The locus of points $\left(x_{i}, y_{i}\right)$ in the $x-y$ plane is a nodal pattern, any line of which can be considered the edge of a membrane, by supposing that a clamp conforming to the line is impressed there.

Solution $W^{(0)}=0$ gives rise to the familiar grid of nodal lines.

Solutions $W^{(n)}=0$, for $n=1,2,3$, and $a=b=1$, have been examined individually. Nine distinct nodal patterns, the result of taking $\alpha=0$ or $\pi / 2$, and $\beta=0$ or $\pi / 2$, are presented in Fig. 1, the fourth pattern of each order being identical with the third upon pattern rotation of $90^{\circ}$ about the origin. In each pattern there is the necessary phase reversal upon crossing a nodal line. However, the finding is that none of these patterns of nodal lines exhibit closed areas within a finite span of membrane; but rather there is amplitude growth without limit as one moves out from the origin parallel to diagonals and between the nodal lines. Hence individual $W^{(n)}$ for $n>0$ are not descriptive of real membranes.

Solutions in combination have received exploratory attention. Solution $W^{(0)}$ perturbed by adding a small proportion of solution $W^{(2)}$ produces a closed boundary that is a smooth distortion of the boundary given by the separable alone. For this combination, the instantaneous displacement is given by

$$
\eta=\left[W^{(0)}+C W^{(2)}\right] \cos \omega t,
$$
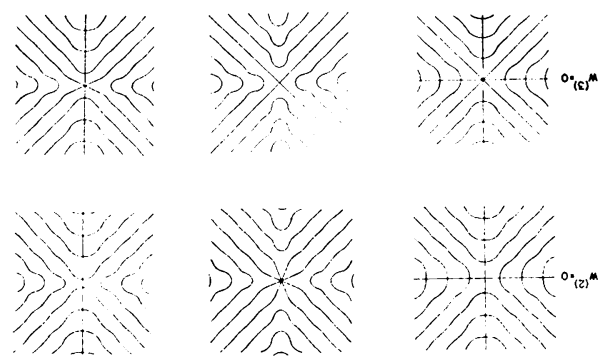

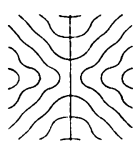

$2,2,:$ :

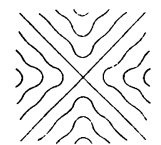

$2 / A: 8$

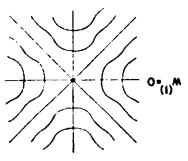

$0: 8$

FIG. 1. Some patterns of $W^{(n)}=0$ on an infinite membrane. 

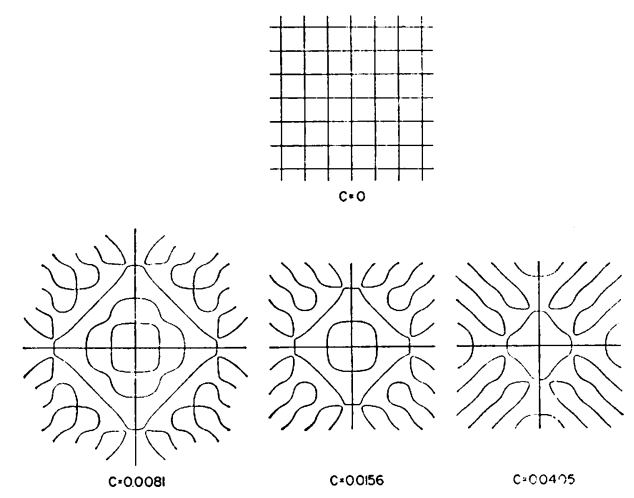

FIG. 2. Nodal patterns on infinite membrane for sums of separable and nonseparable solutions. The function is $W^{(0)}+C W^{(2)}$ with $a=b=1$ and $\alpha=\beta=0$.

where $C$ is a constant and the bracket is the amplitude of the displacement. Zero amplitude occurs at all points $\left(x_{i}, y_{i}\right)$ which solve the two-dimensional transcendental equation

$$
W^{(0)}+C W^{(2)}=0 .
$$

Three evaluations of Eq. (36), with nonzero $C$, have been made, using $\alpha=\beta=0$ and $a=b=1$, the values of $C$ being $C=\left(12.5 \pi^{2}\right)^{-1}=0.0081, C=\left(6.5 \pi^{2}\right)^{-1}=0.0156$, $C=\left(2.5 \pi^{2}\right)^{-1}=0.0405$. The resulting nodal patterns and the familiar nodal pattern for $C=\mathbf{0}$ are presented in Fig. 2.

Several features of the patterns for nonzero $C$ may be noted.

(1) The existence of closed areas shows that nonseparables combined under these conditions should be adaptable to real membranes.

(2) The three patterns suggest (but do not fulfill) an overtone sequence ascending from right to left.

(3) The three patterns, regarded as perturbations of the pattern for $C=0$, are gross distortions of it that leave untouched only the lines that are collinear with the axes.

(4) Most of the nodal lines in the lower three patterns are open to infinity after the manner of the leftmost $W^{(2)}$ pattern in Fig. 1 .

(5) In each pattern there is phase reversal upon crossing any nodal line.

Within closed areas the distribution of amplitude is of interest. Equal amplitude contours have been traced for one of the four closed areas of the pattern for $C=0.0405$ and for one closed area of the pattern for $C=0$. These are presented in Fig. 3. The reference amplitude for both is the center of the square.

The same frequency is associated with each pattern of Figs. 1-3, since all are plotted to the same scale and the frequency of each is governed by Eq. (4) with $a=b$. This frequency is

$$
\omega / 2 \pi=c k / 2 \pi=\sqrt{2} c a / 2 \pi,
$$

where $c$ is the velocity of phase propagation on the membrane. The frequency of resonance of a real membrane depends upon dimension, phase velocity and shape. For either shape in Fig. 3, the frequency is given by the relationship

$$
\omega / 2 \pi=\sqrt{2} c(a S)_{0} / 2 \pi S,
$$

where $S$ is the length of the straight edges of the real membrane and $(a S)_{0}$ is the coordi- 


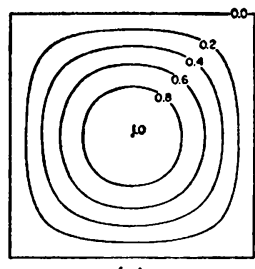

(a)

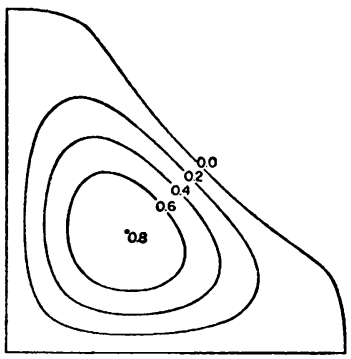

(b)

Fig. 3. Contours of equal amplitude on closed areas selected from patterns for (a) $C=0$ and (b) $C=$ 0.0405 .

nate in radians of the intersection of straight and curved boundaries. The quantity $(a S)_{0}$ is $\pi$ and $1.405 \pi$ in the left and right shapes, respectively, and the side lengths are as 1:1.405.

Figure 4 is a display of membrane shapes and their predicted frequencies expressed in ratio $R$ to that of a square of the same side length. Figure 4 also includes triangle frequencies and interior nodal lines reproduced from other literature [2]. The triangle requires no nonseparable solutions. Arrows in Fig. 4 are intended to show correspondence between shapes of closed areas in the triangles and similar shapes derived from nonseparables.

A final note on the solutions appropriate to finite membranes is that none of the closed contours so far found for $C \neq 0$ is geometrically similar to any other. Therefore the question of producing overtones on a membrane contoured in accordance with any closed area from among the lower three patterns of Fig. 2 is at this time unresolved.

Closed volumes. Closed nodal surfaces were discovered to exist in the bracket of the function

$$
\varphi=\left[W^{(0)}+C W^{(2)}\right] \cos \omega t
$$

when $C=\left(18.75 \pi^{2}\right)^{-1}=.00540$, and $W^{(0)}$ and $W^{(2)}$ are given by Eqs. (22) and (32), respectively, with $a=b=d=1$ and $\alpha=\beta=\delta=0$.
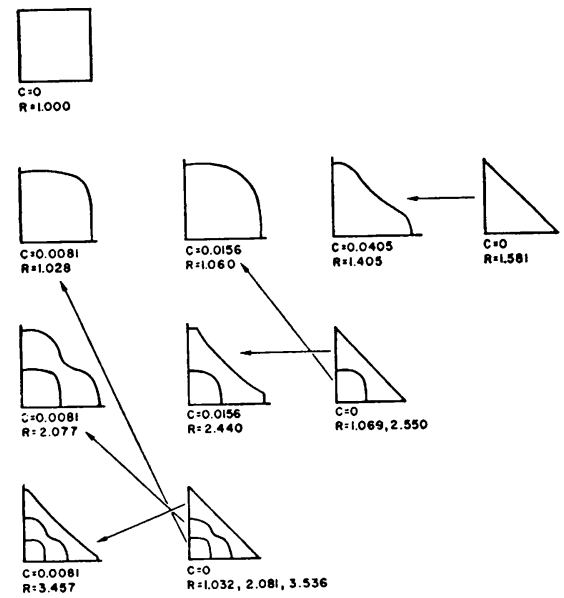

Fig. 4. Closed contours and their frequencies in ratio to that of square of same side length. 

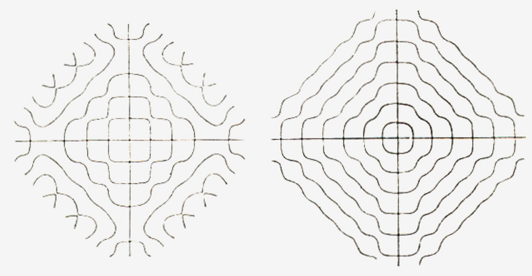

(b)

Fig. 5. Nodal patterns in z-planes passed through closed nodal surfaces. (a) $z= \pm \epsilon$ with $\epsilon \rightarrow 0$ and (b) $z= \pm m \pi$ with $m=1,2,3 \cdots$ The function is $W^{(0)}+.0054 W^{(2)}=0$.

These surfaces are formed by the three planes which contain the coordinate axes and by curved surfaces which meet these planes at right angles. The curved surfaces are the locus of those nonzero points $\left(x_{i}, y_{i}, z_{i}\right)$ which make the bracket of Eq. (39) equal to zero.

The intersection of curved surfaces with the $z$-coordinate plane was found by evaluating points $\left(x_{i}, y_{i}\right)$ for $z=\epsilon$ with $\epsilon$ allowed to approach zero closely enough to justify the approximations $\sin \epsilon=\epsilon$ and $\cos \epsilon=1$. The nodal pattern in the $x-y$ plane given by $z=\epsilon$ is presented in Fig. 5(a). The pattern which is Fig. 5(b) is the intersection of curved nodal surfaces with the planes given by $z= \pm m \pi$ with $m \neq 0$.

Since $W^{(0)}$ and $W^{(2)}$ are symmetrical to cyclic rotation of coordinates, the patterns of Fig. 5 are equally applicable with coordinates cycled. Based upon this property, a three-dimensional model of a portion of one octant was constructed from these patterns as an aid to visualization of the volumes enclosed by the nodal surfaces. Fig. 6 is a reproduction of this model. Wavy lines on the planes are the intersections on them of other nodal surfaces which are apparent in Fig. 5 and which are not closed.

Application to acoustical cavities. In the theory of acoustics the wave equation for sinusoidal waves is Eq. (1), and the boundary condition of an acoustic cavity is a specified wall impedance given by $r m s$ sound pressure in ratio to $r m s$ particle velocity normal to the wall. In symbols the impedance is

$$
Z=p_{r m s} /\left(v_{n}\right)_{r m s},
$$

where $p$ is a scalar and $v_{n}$ is the normal component of the vector particle velocity. Most frequently the assumed condition is that $Z$ is infinite through the vanishing of the normal component of particle velocity upon the wall. The other two of the three mutually

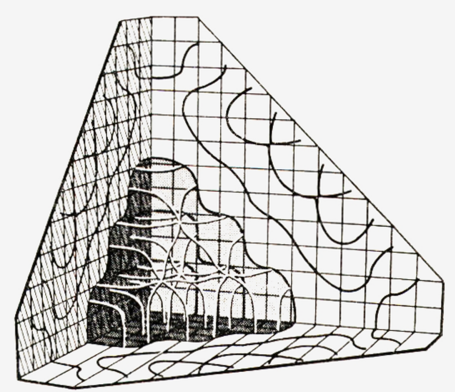

FIG. 6. Three-quarter view of three-dimensional model constructed from nodal patterns of Fig. 5 . Closed volumes are formed by the inner, middle and outer curved surfaces and the halftoned plane regions behind them. 
orthogonal components of particle velocity are not necessarily zero at the wall, and are not determined by that portion of wall.

There has not, as yet, been discovered a nonseparable velocity potential from which to obtain $v_{n}=0$ on a closed surface. There is, however, a mathematical meaning for the alternate condition of zero impedance upon the walls. It is that the pressure there is zero. Thus we can use Eq. (39) to identify $\varphi$ with $p$, or $\varphi$ with a velocity potential from which to derive $p$ according to

$$
p=-\rho \partial \varphi / \partial t
$$

where $\rho$ is the mean mass density of the medium within the cavity.

One may compute frequency of resonance of the volumes of Fig. 6 by referring those volumes to the cubes which would enclose them. The method is essentially that described for membrane patterns. If the frequencies of the distorted cube and of the reference cube be $\omega_{d}$ and $\omega_{r}$, respectively, the ratio $R=\omega_{d} / \omega_{r}$ is as follows: for innermost volume, $R=$ 0.983; inner two volumes, $R=2.042$; all three volumes, $R=3.103$. In each case, the reference cube is one whose side length is equal to the side length of the chosen volume.

While a volume enclosed with walls of zero impedance is mathematically possible, it is nonetheless physically impossible. To produce a pressure-release boundary would require an enclosing wall of no mass or stiffness, yet capable of confining a liquid or gas without escape of matter. Therefore, a practical application of nonseparable solutions to three dimensions is yet to be found.

Summary and conclusions. Sets of nonseparable solutions $W^{(n)}$ with $n>0$ have been defined in two and three rectangular coordinates. Differential operators which generate them from the separable solutions $W^{(0)}$ have also been defined.

The locus of each equation $W^{(n)}=0$, for $n>0$, is a pattern which contains no closed regions of finite area or volume. However, patterns in which finite regions are enclosed have been found for certain two- and three-dimensional renditions of the equation

$$
W^{(0)}+C W^{(2)}=0
$$

in which $C$ is a parametric constant. This means that the left side of Eq. (42) is an eigenfunction for those closed regions. However, an extensive investigation of nonseparables as components of eigenfunctions is needed before applications people can readily use this mathematics.

Acknowledgments. The writer was ably assisted in the performance of this work by J. M. Render of Vitro Laboratories, and he is appreciative of discussions with Drs. Lincoln Bragg, B. R. Levy, and Domina Spencer since the appearance of [1].

Appendix. Reference [1] was written before the term "order" had been chosen or a generative operator had been recognized. It will be helpful to backfit these tools to that paper.

Separable solutions of that paper were Eqs. (2) and (3), which, with order added, are

$$
\begin{aligned}
& A_{r}^{(0)}=\left\{\begin{array}{l}
J_{1}(p r) \\
N_{1}(p r)
\end{array}\right\}\left\{\begin{array}{l}
\cos m z \\
\sin m z
\end{array}\right\} e^{-i \omega t} \\
& A_{z}^{(0)}=\left\{\begin{array}{l}
J_{0}(p r) \\
N_{0}(p r)
\end{array}\right\}\left\{\begin{array}{l}
\sin m z \\
\cos m z
\end{array}\right\} e^{-i \omega t}
\end{aligned}
$$


First and all higher orders can be generated through alternate application of operators

$$
\begin{aligned}
& O_{1}=p \frac{\partial}{\partial m}-m \frac{1}{p} \frac{\partial}{\partial p} p, \\
& O_{2}=p \frac{\partial}{\partial m}-m \frac{\partial}{\partial p},
\end{aligned}
$$

so that

$$
\begin{aligned}
& A_{r}^{(2 n+1)}=O_{1} A_{r}^{(2 n)}, \\
& A_{r}^{(2 n+2)}=O_{2} A_{r}^{(2 n+1)},
\end{aligned}
$$

and

$$
\begin{aligned}
& A_{z}^{(2 n+1)}=O_{2} A_{z}^{(2 n)}, \\
& A_{z}^{(2 n+2)}=O_{1} A_{z}^{(2 n+1)} \quad \text { with } \quad n=0,1,2, \cdots .
\end{aligned}
$$

Equations (7-10) of [1], which contain arbitrary constants $C_{i}$ and $D_{i}$, may be harmonized with the foregoing by setting $C_{2}=C_{1}=-C_{0}=-1, D_{2} p=-D_{1}=$ $-D_{0}=-1$, and by subtracting $2(m / p) A_{z}^{(1)}$ from the $A_{z}$ given by Eq. (10). The sequences become

$$
\begin{aligned}
& A_{r}^{(0)}=J_{1}(p r) \cos (m z) e^{-i \omega t}, \\
& A_{r}^{(1)}=-\left[m r J_{0}(p r) \cos (m z)+p z J_{1}(p r) \sin (m z)\right] e^{-i \omega t}, \\
& A_{r}^{(2)}=-\left\{\left[(p z)^{2}+(m r)^{2}\right] J_{1}(p r) \cos (m z)+p r J_{0}(p r)[\cos (m z)-2 m z \sin (m z)]\right\} e^{-i \omega t},
\end{aligned}
$$

$A_{r}^{(3)}=\cdots$,

and

$$
\begin{aligned}
& A_{z}^{(0)}=J_{0}(p r) \sin (m z) e^{-i \omega t}, \\
& A_{z}^{(1)}=\left[m r J_{1}(p r) \sin (m z)+p z J_{0}(p r) \cos (m z)\right] e^{-i \omega t}, \\
& \begin{aligned}
A_{z}^{(2)}=-\left\{\left[(p z)^{2}+(m r)^{2}+2 m z\right] J_{0}(p r) \cos (m z)\right. \\
\left.\quad-p r J_{1}(p r)[2 m z \cos (m z)+\sin (m z)]\right\} e^{-i \omega t},
\end{aligned}
\end{aligned}
$$

$A_{z}^{(3)}=\cdots$

\section{References}

1. D. S. Moseley, Non-separable solutions of the Helmholtz wave equation, Quart. Appl. Math. 22, 354-357 (1965)

2. P. M. Morse and H. Feshbach, Methods of theoretical physics, McGraw-Hill, New York, 1953, p. 755 